\title{
Faraday rotation effects for diagnosing magnetism in bubble environments
}

\author{
R. Ignace \\ Department of Physics \& Astronomy, East Tennessee State University, Johnson City, USA \\ Correspondence to: R. Ignace (ignace@etsu.edu)
}

Received: 10 February 2014 - Revised: 2 April 2014 - Accepted: 8 April 2014 - Published: 20 May 2014

\begin{abstract}
Faraday rotation is a process by which the position angle (PA) of background linearly polarized light is rotated when passing through an ionized and magnetized medium. The effect is sensitive to the line-of-sight magnetic field in conjunction with the electron density. This contribution highlights diagnostic possibilities of inferring the magnetic field (or absence thereof) in and around wind-blown bubbles from the Faraday effect. Three cases are described as illustrations: a stellar toroidal magnetic field, a shocked interstellar magnetic field, and an interstellar magnetic field within an ionized bubble.
\end{abstract}

\section{Introduction}

Astrophysical magnetism is of broad relevance for understanding the state and history of the universe at a variety of scales, ranging for example from planetary radio emissions (e.g., Grießmeier et al., 2007; Ignace et al., 2010) to interstellar turbulence (e.g., Brandenburg and Lazarian, 2013). It is desirable to have a number of diagnostic approaches for measuring magnetism. One important diagnostic is Faraday rotation, the mechanism through which the line-of-sight (LOS) magnetic field component rotates the position angle (PA) of linear polarization for a beam of radiation. The amount of PA rotation is also proportional to the electron density and the path length through (partially) ionized regions. Additionally, the PA rotation scales with the square of the wavelength of radiation $\lambda^{2}$. Most applications measure the polarization PA for a range of wavelengths to derive the "rotation measure" (or RM) that encodes information about the integrated product of the LOS field component and electron number density (e.g., Draine, 2011). The RM is empirically derived from a logarithmic plot of the polarization PA, $\psi$, against $\lambda^{2}$; the $\mathrm{RM}$ will be the slope of the best-fit line, with RM defined as

$\psi=\left(\frac{e^{3} \lambda^{2}}{2 \pi m_{\mathrm{e}}^{2} c^{4}}\right) \times \int B_{\|} n_{\mathrm{e}} \mathrm{d} z \equiv \mathrm{RM} \times \lambda^{2}$,

where $\lambda$ is the wavelength, $B_{\|}$is the LOS field component in an ionized medium of (position-dependent) electron density $n_{\mathrm{e}}$, and $e, m_{\mathrm{e}}$, and $c$ are fundamental constants in the usual notation (e.g., Draine, 2011).

Many applications of Faraday rotation are for interstellar or extragalactic studies (e.g., Carilli and Taylor, 2002; Han et al., 2006; Beck, 2012). Here emphasis is given to Faraday rotation as a probe of magnetism in and around windblown bubbles. Some recent examples are as follows: Ransom et al. $(2008,2010)$ have conducted studies of Faraday rotation effects arising from planetary nebulae $(\mathrm{PNe})$. Interestingly, Strömgren spheres around white dwarfs may be detectable (e.g., as potentially indicated in Iacobelli et al., 2013). For massive stars: Savage et al. (2013) report on an extensive study of Faraday rotation for the Rosette Nebula HII region. Harvey-Smith et al. (2010) have discovered anti-symmetric PA rotations across the supernova remnant (SNR) G296.5 + 10.0, which they ascribe to the stellar magnetic field in the swept-up wind from a progenitor red supergiant phase. Motivated by these observations, the goal of this contribution is to overview theoretical considerations and tools for interpreting data pertaining to Faraday rotation measurements in wind-blown bubbles. 


\section{Theoretical interpretive tools}

\subsection{Stellar magnetism in wind-blown bubbles}

Consider a rotating star with a stellar wind. Now allow the star to have a magnetic field. A simplified kinematical model, based on the WCFields approach of Ignace et al. (1998), is adopted in which to explore robust Faraday-rotation features that may be observable from radio mapping of the extended stellar wind.

In WCFields the wind is axisymmetric, and the large-scale magnetic field (of relevance to the Faraday effect) is dominated by the toroidal component. For a stellar surface magnetic field initially of strength $B_{*}$ for a star of radius $R_{*}$, the asymptotic toroidal magnetic field will scale as

$B_{\varphi}(r, \vartheta)=B_{*}\left(\frac{v_{\mathrm{rot}}}{v_{\infty}}\right)\left(\frac{R_{*}}{r}\right) \sin \vartheta$,

where $\vartheta$ is the co-latitude of the star, $v_{\infty}$ is the wind terminal speed, and $v_{\text {rot }}$ is the star's equatorial rotation speed. Although not physically self-consistent (see below), for convenience the wind is approximated as spherically symmetric in its density for the results described here, with electron density $n_{\mathrm{e}} \propto r^{-2}$. The assumption of sphericity highlights the influence of the stellar magnetic field on the distribution of Faraday rotation across the bubble.

With spherical density and the above 3-D magnetic topology for a bubble viewed at an inclination angle $i$ to the symmetry axis of the field, the rotation of background linearly polarized light at a fixed wavelength for a sightline intercepting the bubble is given by

$$
\begin{aligned}
\psi_{\text {bub }}(x, y)= & -2 \pi\left(\frac{R_{*}}{z_{\text {bub }}}\right)\left(\frac{y}{\varpi}\right)\left(\frac{R_{*}}{\varpi}\right)^{2} \sin i \\
& \times\left[\left(\frac{\pi}{4}-\frac{\theta_{0}}{2}\right)+\frac{1}{4} \sin 2 \theta_{0}\right] .
\end{aligned}
$$

Here a Cartesian coordinate system $(x, y, z)$ is introduced, with $z$ the observer line-of-sight, and $x$ and $y$ in the plane of the sky, with $x$ along the projected symmetry axis defined by the toroidal field, and then $\hat{y}=\hat{z} \times \hat{x}$. The parameter $\varpi$ is an observer impact parameter, with $\varpi^{2}=x^{2}+y^{2}$. The quantity $\theta_{0}$ is an observer angle for the point at which a sightline intersects the bubble. If the bubble has radius $R_{\text {bub }}$, then $\sin \theta_{0}=\varpi / R_{\text {bub }}$. Finally, $z_{\text {bub }}$ is a scaling factor for the amount of PA rotation by the Faraday effect. It depends on magnetic field strength and the density of the medium. It represents a characteristic length for the PA rotation (see Ignace and Pingel, 2013). Then $\psi_{\text {bub }}$ is the PA rotation integrated along the $z$ coordinate through the bubble at $(x, y)$, or equivlanetly $\varpi$ and azimuth angle. It therefore represents a perturbation to the Faraday rotation as compared to sightlines that pass near the wind bubble but not through it (see Ignace and Pingel, 2013 for a more thorough discussion).

Figure 1 provides a false-color map of $\psi_{\text {bub }}$ based on the preceding equation. Faraday rotation is sensitive to the

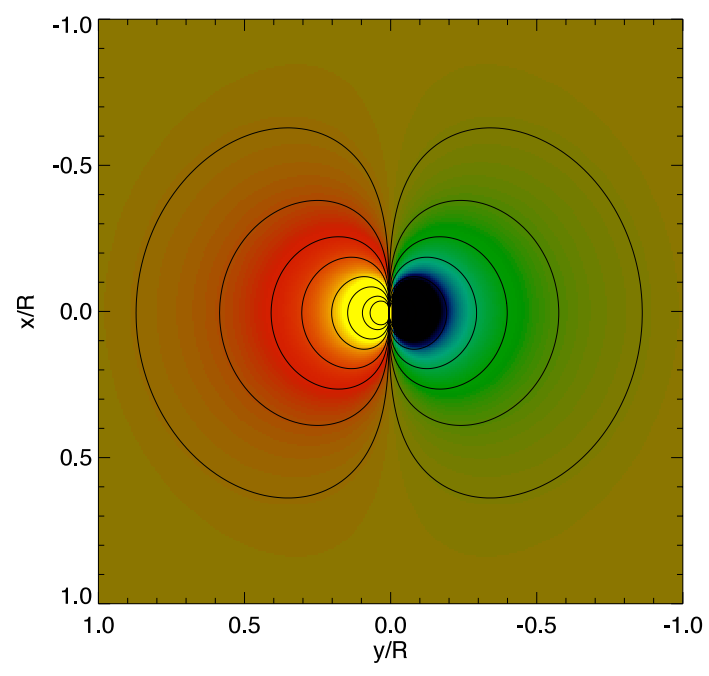

Figure 1. False color image of polarization PA (or alternatively, $\mathrm{RM}$ ) as projected onto the plane of the sky for a model of a windblown bubble threaded by a toroidal stellar magnetic field. Here the opposing colors (blue, green vs. yellow, red) signify a change in polarity of the magnetic field. This is a simulated map of the sky in observer $x, y$ coordinates, normalized to the maximum radial extent of the spherical bubble.

net polarity of the field: the PA rotation is counterclockwise $(R M>0)$ when the field is pointed toward the observer, and clockwise $(\mathrm{RM}<0)$ when opposite. The left-right antisymmetry displayed in Fig. 1 highlights this fact. HarveySmith et al. (2010) used a similar model to interpret such an antisymmetry observed in a SN remnant. Note that for the adopted model, the morphology is independent of the viewing inclination angle, which appears in the solution only as a factor to govern the amplitude for the PA rotation (alternatively, the scale of RM). Although there are ways to improve on this simple model (specifically, allowing for a dynamically self-consistent density distribution, for example axisymmetry following Chevalier and Luo, 1994), antisymmetry in the PA rotation, or equivalently in RM, is a morphologically robust feature of a toroidal magnetic field distribution.

\subsection{Interstellar magnetism in wind-blown bubbles}

Whiting et al. (2009) presented a model for describing the centro-symmetric variation in RM across the face of an HII region under the following circumstances. The spherically symmetric region consists of three zones: (a) an inner nonmagnetic stellar wind as zone 1, (b) an outer region of undisturbed interstellar gas with uniform density and threaded by a uniform interstellar magnetic field as zone 3, and (c) an annular region bounded by an inner contact discontinuity between the stellar wind ${ }^{1}$ and postshocked ISM gas. Except

\footnotetext{
${ }^{1}$ The stellar wind consists of an inner free-streaming wind and a separate annular region of postshocked stellar wind with the contact
} 

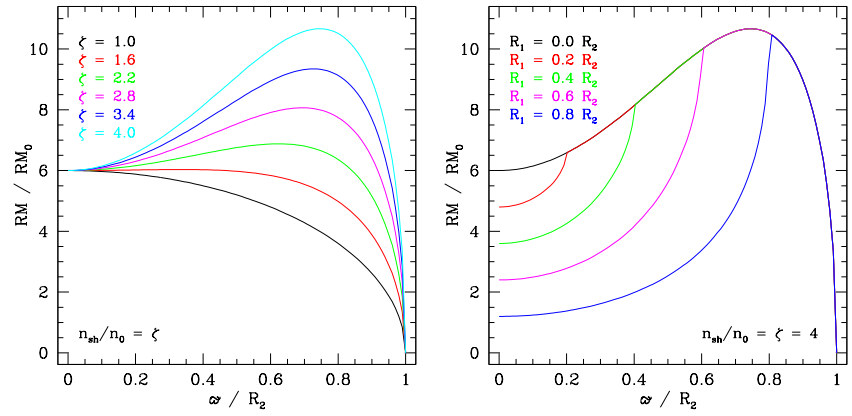

Figure 2. Illustration of the variation of RM with impact parameter across a wind-blown bubble using the Whiting et al. (2009) approach (c.f., Figs. 8 and 9 of Savage et al., 2013). Left shows the RM for different shock strengths $\zeta$ assuming the inner radius $R_{1}=0$. Right shows the case of a fixed shock strength $\zeta=4$ but for different radii $R_{1}$ (see text).

for the magnetic field, the morphology mimics that expected from wind-blown bubbles described in Weaver et al. (1977). The inner field-free zone has radius $R_{1}$ and consists of stellar wind material. Between the radii of $R_{1}$ and $R_{2}$ is the postshocked interstellar gas. Then the pre-shocked interstellar gas exists at radii beyond $R_{2}$.

The interstellar field is taken to be of uniform strength and direction in zone 3, making an angle $i$ to the observer's LOS. For the post-shock magnetic field of zone 2, the shock is approximated as discontinuously enhancing the field component that is perpendicular to the shock normal, $B_{\perp}$, whereas the component of the field parallel to the shock normal, $B_{\|}{ }^{2}$, is unperturbed in its transport across the shock. The strength of the post-shock component $B_{\perp}$ depends on the shock strength $\zeta \equiv n_{\text {sh }} / n_{0}$, with $n_{\text {sh }}$ the post-shock gas number density, $n_{0}$ the pre-shock interstellar gas number density, and $1 \leq \zeta \leq 4$.

A simplification adopted by Whiting et al. (2009) is that for a sightline passing through zone 2 , the post-shock vector field at the back boundary is considered constant for the back half of the pathlength, and the post-shock vector field at the front boundary is likewise constant on the front half of the path. This approximation provides an analytic solution for the RM distribution (equivalent to PA rotations) across the face of the bubble. And as in the preceding section, the simplification leads to solutions that contain robust elements that will prove useful for evaluating more elaborate models.

discontinuity as its outer boundary. These distinct divisions for the stellar wind region are not separately indicated in the figure since the entirety of wind is assumed non-magnetic and so is not relevant to the Faraday rotation in this model.

${ }^{2}$ One should not confuse the parallel field component in relation to the shock normal with the parallel component along the LOS that is relevant to Faraday rotation.
Although the model is based on Whiting et al. (2009), a different notation and form of expression are used here. The impact parameter is again given by $\varpi$. Then the radius for any point in the bubble is $r^{2}=\varpi^{2}+z^{2}$. The solution for RM arising from zone 2 of the bubble alone, in terms of the angle $\theta$, where $\sin \theta=\varpi / R_{2}$, is

$$
\mathrm{RM} \propto \cos \theta\left(\zeta \sin ^{2} \theta+\cos ^{2} \theta\right)\left[1-a(\theta) H\left(\theta-\theta_{\mathrm{c}}\right)\right] .
$$

Here, $H(u)$ is the Heaviside function, defined so that $H=$ 0 when the argument of the function $u<0$, and $H=1$ for $u \geq 0$. The critical angle $\theta_{\mathrm{c}}$ is $\sin \theta_{\mathrm{c}}=R_{1} / R_{2}$. For $\theta<\theta_{\mathrm{c}}$, a sightline intersects zone 1 ; for $\theta \geq \theta_{\mathrm{c}}$, it does not. Finally, the function $a$ is a path length correction for those sightlines that cross zone 1 , with

$a(\theta)=\frac{\sqrt{R_{1}^{2}-\varpi^{2}(\theta)}}{\sqrt{R_{2}^{2}-\varpi^{2}(\theta)}}$.

The form of Eq. (4) explicitly assumes constant density and uniform ionization throughout zone 2 .

Example profiles for how the RM varies with impact parameter are displayed in Fig. 2. The left panel displays a limiting case with $R_{1}=0$; the curves are for different shock strengths. The right panel is for a strong shock with $\zeta=4$ fixed, and $R_{1}$ is allowed to vary. Note that for $\zeta=4$, introducing $R_{1}$ amounts to removing varying portions from the top curve in the left panel depending on the extent of $R_{1}$. This results in a discontinous change of slope in RM where $\varpi=R_{1}$.

Although the details of the RM profile will change if this model is made more realistic, the appearance of the discontinuous change in slope is a geometric effect and should be a fairly robust feature of models that have the characteristic three-zone structure used in Whiting et al. (2009). Further refinements to the Whiting et al. (2009) model will ultimately be driven by the data. In an application to the Rosette Nebula (Savage et al., 2013), the model in its present form appears adequate; however, the sampling of sightlines is sparse at low impact parameters so that some model parameters are not well-constrained. As better data become available, the two most likely features to be improved will be: first the variation of the vector field throughout zone 2, and second accounting for departures from spherical symmetry (an axisymmetric bubble would be more realistic). Moreover, should one observe that a profile solution provides an overall match to the general contour of the data, but that the data display considerable dispersion, this could signify the presence of a random field component. A stochastic contribution to the magnetic field distribution can arise because of turbulence. 


\subsection{Interstellar magnetism in an ionized bubble}

As a final example, consider a spherical bubble in which the only effect is that the region is more ionized, relative to the local interstellar gas, by the central UV-bright star. In this case there is no interaction with a stellar wind over most of the ionized bubble, and the gas is permeated solely by the pre-existing interstellar magnetic field. Generally, the gas is turbulent. Often the field is conveniently approximated as consisting of two components: a globally uniform component and a locally random one.

It is interesting first to consider a purely uniform field in the ionized bubble. This field everywhere has the same LOS component toward the observer. However, sightlines through the spherical bubble depend on the impact parameter, with path length $\Delta z=2 \sqrt{R_{\text {bub }}^{2}-\varpi^{2}}$. For a constant density, the effect of the bubble for PA rotations is a function of path length only, with $\psi_{\text {bub }} \propto \Delta z$. If one were to produce a map of polarization PA, it would be seen to be centro-symmetric. One could construct a histogram of the incidence of $\psi$ values as a function of impact parameter. Theoretically, for constant density and a uniform field, one expects the incidence of different PA rotations across the bubble $(d \mathcal{N} / d \psi)$ bub to be

$$
\left(\frac{\mathrm{d} \mathcal{N}}{\mathrm{d} \psi}\right)_{\text {bub }} \propto \varpi\left(\frac{\mathrm{d} \varpi}{\mathrm{d} \psi}\right)_{\text {bub }} \propto \psi_{\text {bub }}
$$

Consequently, a histogram for the incidence of $\psi_{\text {bub }}$ values is linear in $\psi_{\text {bub itself. }}$

This is important because it means that deviations from linearity in such a construction, which is a rather convenient one for observers to make, imply a deviation from one or more of the underlying assumptions. In the case of a bubble that is not perfectly spherical in shape, one might produce a mild alteration to the linear distribution $d \mathcal{N} / \psi$ expected from exact sphericity. Something similar would result if the field were not perfectly uniform, but slowly varying in strength or direction throughout the bubble volume. On the other hand, stochastic variations in density and/or the magnetic field (in strength or direction) could produce sharp features in the distribution or perhaps increase the dispersion in histogram values. A histogram of the PA measures (or RM values) represents a moment of the data, and may be used constructively in conjunction with the image itself to aid in the interpretation of polarimetric imaging data in and around ionized bubbles.

\section{Conclusions}

There are opportunities for exploring stellar and interstellar magnetism in the vicinity of wind-blown bubbles. One can think of the bubbles as perturbations that modify the Faraday rotation for sightlines that intercept a bubble as compared to neighboring sightlines that do not, leading to a RM anomaly, a term introduced by Whiting et al. (2009). By compar- ing the observed variations in the PA of the diffuse emission across a bubble to models surrounding ISM (e.g., magnetic field geometry, electron distribution, shock strength) can be extracted. Since PAs (or likewise RMs) are obtained by comparing sightlines on and off the source, mapping at just one wavelength is sufficient for thi strategy. On the other hand, some studies, like that of the Rosette by Savage et al. (2013), make use of distant polarized point sources to map out Faraday rotation across a bubble in a "buckshot" approach. This can be advantageous if the diffuse background is non-uniform, but requires mapping at multiple wavelengths, since the unrotated PAs of the distant unrelated and independent sources are not known. Although analysis for Faraday rotation can be challenging, the underlying physics provides tremendous diagnostic potential for studying the environments of circumstellar and interstellar magnetized plasmas.

Acknowledgements. Ignace expresses appreciation to Steve Spangler and two anonymous referees for providing several helpful comments to improve this contribution. Support for this research through a grant from the National Science Foundation (AST-0807664) is gratefully acknowledged.

Edited by: H. Fichtner

Reviewed by: two anonymous referees

\section{References}

Beck, R.: Magnetic Fields in Galaxies, Space Sci. Rev., 166, 215330, doi:10.1007/s11214-011-9782-z, 2012.

Brandenburg, A. and Lazarian, A.: Astrophysical Hydromagnetic Turbulence, Space Sci. Rev., 178, 163-200, doi:10.1007/s11214013-0009-3, 2013.

Carilli, C. L. and Taylor, G. B.: Cluster Magnetic Fields, Annu. Rev. Astron. Astr., 40, 319-348, doi:10.1146/annurev.astro.40.060401.093852, 2002.

Chevalier, R. A. and Luo, D.: Magnetic shaping of planetary nebulae and other stellar wind bubbles, Astrophys. J., 421, 225-235, doi:10.1086/173640, 1994.

Draine, B. T.: Physics of the Interstellar and Intergalactic Medium, Princeton University Press, USA, 2011.

Grießmeier, J.-M., Zarka, P., and Spreeuw, H.: Predicting lowfrequency radio fluxes of known extrasolar planets, Astron. Astrophys., 475, 359-368, doi:10.1051/0004-6361:20077397, 2007.

Han, J. L., Manchester, R. N., Lyne, A. G., Qiao, G. J., and van Straten, W.: Pulsar Rotation Measures and the Large-Scale Structure of the Galactic Magnetic Field, Astrophys. J., 642, 868-881, doi:10.1086/501444, 2006.

Harvey-Smith, L., Gaensler, B. M., Kothes, R., Townsend, R. Heald, G. H., Ng, C.-Y., and Green, A. J.: Faraday Rotation of the Supernova Remnant G296.5+10.0: Evidence for a Magnetized Progenitor Wind, Astrophys. J., 712, 1157-1165, doi: 10.1088/0004637X/712/2/1157, 2010.

Ignace, R., Cassinelli, J. P., and Bjorkman, J. E.: "WCFields": A Magnetic Rotating Stellar Wind Model from Wind Compression Theory, Astrophys. J., 505, 910-920, doi:10.1086/306189, 1998. 
Ignace, R., Giroux, M. L., and Luttermoser, D. G.: Radio emissions from substellar companions of evolved cool stars, Mon. Not. R. Astron. Soc., 402, 2609-2616, doi:10.1111/j.13652966.2009.16085.x, 2010.

Iacobelli, M., Haverkorn, M., and Katgert, P.: Rotation measure synthesis at the $2 \mathrm{~m}$ wavelength of the FAN region: unveiling screens and bubbles, Astron. Astrophys., 549, 56, doi:10.1051/0004-6361/201220175, 2013.

Ignace, R. and Pingel, N.: Faraday Rotation Distributions from Stellar Magnetism in Wind-blown Bubbles, Astrophys. J., 765, 19, doi:10.1088/0004-637X/765/1/19, 2013.

Ransom, R. R., Uyaniker, B., Kothes, R., and Landecker, T. L.: Probing the Magnetized Interstellar Medium Surrounding the Planetary Nebula Sh 2-216, Astrophys. J., 684, 1009-1017, doi:10.1086/590656,2008.
Ransom, R. R., Kothes, R., Wolleben, M., and Landecker, T. L.: Faraday Rotation in the Tail of the Planetary Nebula DeHt 5, Astrophys. J., 724, 946-956, doi:10.1088/0004-637X/724/2/946, 2010.

Savage, A. H., Spangler, S. R., and Fischer, P. D.: Probing the Rosette Nebula Stellar Bubble with Faraday Rotation, Astrophys. J., 765, 42, doi:10.1088/0004-637X/765/1/42, 2013.

Weaver, R., McCray, R., Castro, J., Shapiro, P., and Moore, R.: Interstellar bubbles. II - Structure and evolution, Astrophys. J., 218, 377-395, doi:10.1086/155692, 1977.

Whiting, C. A., Spangler, S. R., Ingleby, L. D., and Haffner, L. M.: Confirmation of a Faraday Rotation Measure Anomaly in Cygnus, Astrophys. J., 694, 1452-1463, doi:10.1088/0004637X/694/2/1452, 2009. 